\title{
Pesticide residues screening in wine by mass spectrometry
}

\author{
Andrea F. Machado, Suelen Militão, Magda M. Sarubbi, and Alexandre Brandelli
}

NSF Bioensaios, 94480-775 Viamão, Brazil

\begin{abstract}
Recently, a study (from PAN Europe) covered 40 bottles of wine - 34 conventional and six organic ones - purchased inside the EU. According to the results, the 34 bottles of conventional wine together contained 148 pesticide residues. All 34 bottles contained from one to ten pesticides, bringing the average per bottle to more than four. Of the six bottles of organic wine tested, one sample contained a low concentration of a possibly carcinogenic pesticide. According to PAN Europe, the "contamination of wines is a direct result of over-reliance on pesticides in grape production". This study, between others, to prove the importance of develop methods sensivity and confident for pesticide detection in wine. A multiresidue method was developed for the determination ca of 250 pesticide residues in wine using Quechers extraction, gas chromatography-tandem mass spectrometry (GC-MS-MS) and liquid chromatography-tandem mass spectrometry (LC-MS-MS). The method was validated with the evaluation of follow parameters: Linearity, Precision, Accuracy, Matrix effect, Limit of detection and Limit of Quantification. The method was approved and was able to quantify pesticide residues in more than 60 samples of wine.
\end{abstract}

\section{Introduction}

In this paper, we report a rapid modified QuEChERS method for multiresidue analysis for ca of 300 pesticides in wine with good selectivity, sensitivity, and cost effectiveness. In order to demonstrate the suitability of the method for routine regulatory purposes, the method was validated and the statistical parameters are discussed.

\section{Experimental}

\subsection{Reagents and standards}

The certificated analytical standards were purchased from Dr. Ehrenstorfer GmbH (Augsburg, Alemanha). All the solvents and chemicals used in the study were of HPLC grade.

\subsection{Instrumentation}

Ultra high-performance liquid chromatography UHPLCMS/MS (liquid chromatography, Shimadzu and MSMS, API 4000 Applied Biosystems) equipped with Phenomenex Kinetex column $(50 \mathrm{~mm} \times 2.1 \mathrm{~mm}, 2.6 \mu \mathrm{m})$ was used for quantification of pesticides. The flow rate was $250 \mu \mathrm{L} / \mathrm{min}$, the column temperature $30^{\circ} \mathrm{C}$, and the injection volume $5 \mu \mathrm{L}$. A binary gradient of $0.1 \% \mathrm{HCOOH}$ and $\mathrm{HCOONH} 5 \mathrm{mM}$ in water (A) and $0.1 \% \mathrm{HCOOH}$ and $\mathrm{HCOONH} 5 \mathrm{mM}$ in $\mathrm{CHOH}$ (B) was employed. The mobile-phase gradient was programmed as follows: $0 \mathrm{~min}$, $20 \% \mathrm{~B} ; 10 \mathrm{~min}, 80 \% \mathrm{~B} ; 10.01 \mathrm{~min}, 90 \% \mathrm{~B} ; 13 \mathrm{~min}, 90 \%$ B; $13.01 \mathrm{~min}, 20 \% \mathrm{~B}$; and $16 \mathrm{~min}, 20 \% \mathrm{~B}$. Mass spectral analyses were operated in the positive ion mode using a ESI interface. The electrospray ionization (ESI) needle spray voltage was 5000 . The heated capillary was $500^{\circ} \mathrm{C}$. Collision Gas Pressure was 5. The pesticides were detected in MS/MS conditions, programming the chromatographic run in MRM mode (multiple reaction monitoring).

\subsection{Method validation}

\subsubsection{Specificity}

The specificity of the analytical method for pesticides detection was confirmed by obtaining positive results from honey containing the analyte, coupled with negative results from samples which do not contain it (negative controls). The matrix effect was assessed by preparing pesticide standards in blank matrix extracted from untreated honey. The matrix extracts were analyzed before spiking to confirm the absence of the test pesticides in them.

\subsubsection{Linearity}

The quantification of pesticide was based on a six-point matrix-matched calibration graph by plotting the detector response against concentration of the calibration standards within the range $2.5-50 \mu \mathrm{g} / \mathrm{L}$ making three replicates for each concentration. A linear regression of six calibration points for each component was used to determine the relationship with the analyte concentrations calculated for each component on the basis of their occurrence in the reference material.

\subsubsection{Limit of Detection (LOD) and Limit of Quantification (LOQ)}

The LOD and LOQ were determined by signal-to-noise approach.

\subsubsection{Method Accuracy (Recovery) and Precision (Repeatability)}

Method recovery studies were performed at two spiking concentration levels (10 $\mu \mathrm{g} / \mathrm{kg}$ and $50 \mu \mathrm{g} / \mathrm{kg})$. 


\subsubsection{Determination of uncertainties}

Combined uncertainty in estimation was determined for all the pesticides at the two fortification levels studied (10 and $50 \mu \mathrm{g} / \mathrm{kg}$ ) as the statistical procedure of the EURACHEM/CITAC Guide CG 4.

\section{Results and discussion}

\subsection{Method validation}

\subsubsection{Specificity}

The specificity of the method toward the studied analytes was good. No interferences due to matrixes were found. Hence, no further time-consuming concentration/cleanup pretreatments were required.

\subsubsection{Linearity}

The analytes showed linear behavior in the studied concentration range of $2,5-50 \mu \mathrm{g} / \mathrm{L}$. The correlation coefficient $\left(\mathrm{r}^{2}\right)$ was found to be $\geq 0.99$ for all pesticides.

\subsection{3. $L O D$ and $L O Q$}

LOD and LOQ were estimated as the lowest concentrations of pesticide injected that yielded a signal/noise ratio of 3 and 10, respectively. The LOQs attained in the proposed method fit with maximum residue limits (MRLs) for grape. The limits were reported in Table 1.

\subsubsection{Recovery and precision}

The single-step extraction method adopted for wine samples provided satisfactory recovery. These data are in agreement with the criteria of document no. SANCO/12495/2011, that recommend general recovery limits of $70-120 \%$ within laboratory repeatability $\leq 20 \%$ [18]. Therefore, the method could be considered sufficiently accurate and precise for the purpose.

\subsubsection{Uncertainty of measurement}

The study of uncertainty was performed at 2 concentration levels (10 and $50 \mu \mathrm{g} / \mathrm{kg}$ ), identifying and studying the most important parameters that determined the uncertainty of the analytical method. The parameters selected were point calibration, standard solution, weigh, volume, and precision; their contributions to method uncertainty were calculated as indicated in the experimental section. The different contributions of uncertainty for each concentration level, together with the relative combined standard uncertainty, are shown in Table 1.

\subsection{Wine samples analysis}

Sixty wine samples were analyzed between 2012 and 2015. The samples were from different wineries in the state of Rio Grande do Sul, Brazil. About 300 pesticides were analyzed and 30 were detected. 40 samples were determined at least one pesticide.

\section{Conclusion}

Based on these results, the methodology for the determination of pesticides in wine, described in this report, are validated and approved for execution.
Table 1. Results for LOQ, Recovery, Precision and Uncertainty of Measurement.

\begin{tabular}{|c|c|c|c|c|}
\hline Pesticide & LOQ & Recovery & SD\% & UM \\
\hline 3OHCarbofurano & 0.01 & 76.62 & 3.41 & 0.0006 \\
\hline Acefato & 0.01 & 82.82 & 6.78 & 0.0014 \\
\hline Acetamiprid & 0.01 & 79.63 & 3.84 & 0.0008 \\
\hline Acrinatrin & 0.01 & 86.37 & 7.00 & 0.0015 \\
\hline Aldicarb & 0.01 & 84.07 & 5.43 & 0.001 \\
\hline AldicarbSulfone & 0.01 & 78.10 & 4.83 & 0.0009 \\
\hline AldicarbSulfoxide & 0.01 & 98.26 & 3.56 & 0.0009 \\
\hline Aletrina & 0.01 & 73.38 & 4.31 & 0.0008 \\
\hline Ametrina & 0.01 & 76.66 & 4.10 & 0.0008 \\
\hline Amicarbazone & 0.01 & 86.39 & 6.91 & 0.0015 \\
\hline Azaconazole & 0.01 & 96.63 & 4.33 & 0.0010 \\
\hline AzinfosEtil & 0.01 & 84.89 & 6.80 & 0.001 \\
\hline AzinfosMetil & 0.01 & 86.38 & 6.58 & 0.0014 \\
\hline Azoxistrobin & 0.02 & 84.91 & 11.80 & 0.0025 \\
\hline Benalaxil & 0.01 & 85.57 & 6.10 & 0.0013 \\
\hline Benfluralina & 0.24 & 93.80 & 18.62 & 0.0249 \\
\hline Benfurocarb & 0.02 & 97.09 & 8.73 & 0.0021 \\
\hline Bifentrina & 0.01 & 81.91 & 3.68 & 0.0007 \\
\hline Bioaletrina & 0.02 & 84.25 & 10.30 & 0.0022 \\
\hline Bitertanol & 0.01 & 97.89 & 5.95 & 0.0014 \\
\hline Boscalide & 0.02 & 87.39 & 9.69 & 0.0021 \\
\hline Bupirimato & 0.01 & 91.68 & 5.13 & 0.0012 \\
\hline Buprofezin & 0.01 & 85.86 & 6.13 & 0.0013 \\
\hline Cadusafos & 0.01 & 82.20 & 3.55 & 0.0007 \\
\hline Carbaril & 0.01 & 82.87 & 7.27 & 0.0015 \\
\hline Carbendazim & 0.01 & 111.80 & 4.04 & 0.0011 \\
\hline Carbofenotion & 0.01 & 94.27 & 5.32 & 0.0012 \\
\hline Carbofurano & 0.01 & 85.51 & 5.19 & 0.0011 \\
\hline Carboxim & 0.01 & 82.59 & 5.15 & 0.0011 \\
\hline Carpropamida & 0.01 & 86.65 & 6.33 & 0.0014 \\
\hline CialofopPbutil & 0.01 & 87.27 & 6.67 & 0.0014 \\
\hline Cianofenfos & 0.01 & 81.22 & 6.47 & 0.0013 \\
\hline Cifenotrina & 0.04 & 85.22 & 3.81 & 0.0040 \\
\hline Cimoxamil & 0.01 & 88.61 & 5.28 & 0.0012 \\
\hline Cipermetrina & 0.05 & 86.90 & 5.24 & 0.0056 \\
\hline Ciproconazol & 0.03 & 85.41 & 13.68 & 0.0029 \\
\hline Ciprodinil & 0.01 & 86.62 & 4.24 & 0.0009 \\
\hline Cletodim & 0.01 & 102.43 & 5.64 & 0.0014 \\
\hline Clorfentezine & 0.01 & 88.26 & 6.35 & 0.0014 \\
\hline Clorfenvinfos & 0.01 & 85.74 & 7.27 & 0.0015 \\
\hline Clorfluazuron & 0.01 & 92.12 & 5.56 & 0.0013 \\
\hline ClorimuromEtil & 0.01 & 94.75 & 6.04 & 0.0014 \\
\hline Cloroprofan & 0.01 & 88.52 & 6.92 & 0.0015 \\
\hline Clorpirifos & 0.01 & 106.75 & 3.73 & 0.0010 \\
\hline ClorpirifosMetil & 0.01 & 91.66 & 5.27 & 0.0012 \\
\hline Clortiofos & 0.01 & 90.46 & 6.04 & 0.0014 \\
\hline Clotianidina & 0.01 & 76.64 & 5.96 & 0.0011 \\
\hline Coumofos & 0.01 & 90.80 & 5.01 & 0.0011 \\
\hline Cyazofamide & 0.01 & 92.42 & 3.79 & 0.0009 \\
\hline Cyflutrina & 0.25 & 94.80 & 15.75 & 0.0254 \\
\hline Cyromazine & 0.01 & 79.56 & 5.54 & 0.0011 \\
\hline
\end{tabular}


Table 1. Continued.

\begin{tabular}{|c|c|c|c|c|}
\hline Pesticide & LOQ & Recovery & SD\% & UM \\
\hline Dazomete & 0.01 & 91.45 & 5.28 & 0.0012 \\
\hline Deltametrina & 0.06 & 87.60 & 5.28 & 0.0057 \\
\hline DemetonSmetil & 0.01 & 88.41 & 4.28 & 0.0009 \\
\hline Diazinon & 0.01 & 84.40 & 6.56 & 0.0014 \\
\hline Diclofluanid & 0.01 & 88.32 & 5.89 & 0.0013 \\
\hline DiclofopMetil & 0.01 & 80.41 & 5.56 & 0.0011 \\
\hline Dicloran & 0.10 & 85.80 & 10.16 & 0.0108 \\
\hline Diclorvos & 0.01 & 102.42 & 4.25 & 0.0011 \\
\hline Dicrotofos & 0.01 & 74.14 & 4.38 & 0.0008 \\
\hline Difenoconazol & 0.01 & 80.32 & 3.25 & 0.0006 \\
\hline Diflubenzuron & 0.01 & 88.69 & 6.60 & 0.0015 \\
\hline Dimetoato & 0.01 & 95.07 & 4.37 & 0.0010 \\
\hline Dimetomorf & 0.02 & 97.24 & 8.48 & 0.0020 \\
\hline Diniconazol & 0.01 & 92.81 & 5.23 & 0.0012 \\
\hline Dinocap & 0.01 & 75.90 & 5.70 & 0.0011 \\
\hline Dinoterb & 0.01 & 93.88 & 2.56 & 0.0006 \\
\hline DisulfotonSulfone & 0.01 & 89.91 & 5.39 & 0.0012 \\
\hline DisulfotonSulfoxide & 0.01 & 80.64 & 6.05 & 0.0012 \\
\hline Diuron & 0.02 & 88.79 & 7.18 & 0.0016 \\
\hline Dodemorf & 0.01 & 93.90 & 4.83 & 0.0011 \\
\hline EPN & 0.01 & 89.27 & 3.35 & 0.0007 \\
\hline Epoxiconazol & 0.01 & 87.79 & 5.77 & 0.0013 \\
\hline Etiofencarb & 0.01 & 89.59 & 4.81 & 0.0011 \\
\hline EtiofencarbSulfone & 0.01 & 88.13 & 5.54 & 0.0012 \\
\hline EtiofencarbSulfoxide & 0.01 & 89.07 & 6.95 & 0.0015 \\
\hline Etion & 0.01 & 81.64 & 5.31 & 0.0011 \\
\hline Etiprole & 0.01 & 87.00 & 4.29 & 0.0009 \\
\hline Etofenprox & 0.01 & 78.08 & 7.43 & 0.0014 \\
\hline Etoprofos & 0.01 & 79.45 & 6.05 & 0.0012 \\
\hline Etoxisulfuron & 0.01 & 77.72 & 7.66 & 0.0015 \\
\hline Etrinfos & 0.01 & 89.74 & 6.84 & 0.0015 \\
\hline Famoxadone & 0.02 & 91.24 & 7.93 & 0.0018 \\
\hline Fenamidone & 0.01 & 92.50 & 6.59 & 0.0015 \\
\hline Fenamifos & 0.01 & 87.67 & 6.66 & 0.0014 \\
\hline FenamifosSulfone & 0.01 & 96.77 & 3.17 & 0.0008 \\
\hline FenamifosSulfoxide & 0.01 & 87.90 & 7.01 & 0.0015 \\
\hline Fenarimol & 0.02 & 88.04 & 9.60 & 0.0021 \\
\hline Fenazequin & 0.01 & 92.67 & 5.14 & 0.0012 \\
\hline Fenbuconazol & 0.01 & 91.22 & 4.37 & 0.0010 \\
\hline fenhexamid & 0.01 & 87.00 & 5.23 & 0.0011 \\
\hline FenoxapopPetil & 0.01 & 100.12 & 3.14 & 0.0008 \\
\hline Fenpiroximate & 0.01 & 87.49 & 5.25 & 0.0011 \\
\hline Fenpoprimorf & 0.01 & 80.88 & 3.21 & 0.0006 \\
\hline Fenpropatrina & 0.04 & 87.74 & 3.68 & 0.0040 \\
\hline Fention & 0.01 & 80.57 & 7.30 & 0.0015 \\
\hline FentionSulfone & 0.01 & 88.90 & 5.67 & 0.0012 \\
\hline FentionSulfoxide & 0.01 & 92.70 & 4.48 & 0.0010 \\
\hline Fenvalerato & 0.02 & 74.26 & 2.46 & 0.0023 \\
\hline Fipronil & 0.01 & 82.35 & 6.81 & 0.0014 \\
\hline FipronilSulfone & 0.01 & 103.10 & 4.31 & 0.0011 \\
\hline Flazasulfuron & 0.01 & 95.33 & 2.75 & 0.0006 \\
\hline Fluazifop & 0.01 & 82.32 & 6.58 & 0.0013 \\
\hline
\end{tabular}

Table 1. Continued.

\begin{tabular}{|c|c|c|c|c|}
\hline Pesticide & LOQ & Recovery & SD\% & UM \\
\hline Fludioxonil & 0.01 & 87.36 & 6.50 & 0.0014 \\
\hline Flufenacet & 0.01 & 85.77 & 6.10 & 0.0013 \\
\hline Flufenoxuron & 0.01 & 91.17 & 6.37 & 0.0014 \\
\hline Fluquinconazol & 0.01 & 96.14 & 4.23 & 0.0010 \\
\hline Flusilazole & 0.01 & 87.85 & 6.01 & 0.0013 \\
\hline Flutriafol & 0.01 & 88.88 & 5.57 & 0.0012 \\
\hline Folpete & 0.02 & 89.51 & 7.82 & 0.0017 \\
\hline Fostiazate & 0.01 & 78.64 & 5.09 & 0.0010 \\
\hline Furatiocarb & 0.01 & 82.58 & 5.54 & 0.0011 \\
\hline heptenofos & 0.01 & 85.87 & 5.55 & 0.0012 \\
\hline Hexaconazol & 0.01 & 80.70 & 5.64 & 0.0011 \\
\hline Hexazinona & 0.01 & 80.06 & 3.16 & 0.0006 \\
\hline Hexitiazox & 0.01 & 83.57 & 5.86 & 0.0012 \\
\hline Imazalil & 0.01 & 84.93 & 5.82 & 0.0012 \\
\hline Imazapir & 0.01 & 106.60 & 3.98 & 0.0011 \\
\hline Imibenconazole & 0.01 & 90.69 & 5.74 & 0.0013 \\
\hline Imidacloprid & 0.02 & 86.34 & 8.95 & 0.0019 \\
\hline Indoxacarb & 0.02 & 88.54 & 9.08 & 0.0020 \\
\hline Iprovaliocarb & 0.01 & 84.64 & 6.24 & 0.0013 \\
\hline Isocarbofos & 0.00 & 94.30 & 1.63 & 0.0004 \\
\hline Isoxaflutole & 0.01 & 84.04 & 7.09 & 0.0015 \\
\hline KresoximMetil & 0.02 & 83.10 & 7.85 & 0.0016 \\
\hline LambdaCialotrina & 0.04 & 84.32 & 3.69 & 0.0039 \\
\hline Linuron & 0.01 & 88.29 & 4.74 & 0.0010 \\
\hline Lufenuron & 0.01 & 84.25 & 6.33 & 0.0013 \\
\hline Malaoxon & 0.01 & 81.94 & 4.80 & 0.0010 \\
\hline Malation & 0.01 & 93.48 & 6.24 & 0.0014 \\
\hline Mepanipirim & 0.01 & 88.54 & 6.30 & 0.0014 \\
\hline Mesotrione & 0.02 & 71.01 & 10.86 & 0.0019 \\
\hline Metalaxil & 0.02 & 87.78 & 11.02 & 0.0024 \\
\hline Metamidofos & 0.01 & 74.31 & 4.31 & 0.0008 \\
\hline Meticonazol & 0.01 & 79.50 & 6.44 & 0.0013 \\
\hline Metidation & 0.01 & 89.60 & 6.81 & 0.0015 \\
\hline Metiocarb & 0.01 & 86.63 & 6.96 & 0.0015 \\
\hline MetiocarbSulfone & 0.01 & 91.07 & 3.77 & 0.0009 \\
\hline MetiocarbSulfoxide & 0.002 & 96.04 & 0.97 & 0.0002 \\
\hline Metobromuron & 0.01 & 90.39 & 5.71 & 0.0013 \\
\hline Metomil & 0.01 & 79.63 & 5.63 & 0.0011 \\
\hline Metoxifenozide & 0.02 & 78.95 & 8.88 & 0.0017 \\
\hline Metribuzim & 0.02 & 87.65 & 7.51 & 0.0016 \\
\hline MetsulfuronMetil & 0.01 & 82.32 & 6.16 & 0.0013 \\
\hline Mevinfos & 0.01 & 76.26 & 4.34 & 0.0008 \\
\hline Miclobutanil & 0.02 & 90.17 & 10.15 & 0.0023 \\
\hline Molinato & 0.01 & 95.76 & 5.22 & 0.0012 \\
\hline Monocrotofos & 0.01 & 74.38 & 6.73 & 0.0012 \\
\hline Monuron & 0.01 & 83.13 & 3.87 & 0.0008 \\
\hline Napronamide & 0.01 & 87.56 & 6.98 & 0.0015 \\
\hline Nitenpiram & 0.01 & 103.73 & 3.80 & 0.0010 \\
\hline Nuarimol & 0.01 & 88.85 & 6.78 & 0.0015 \\
\hline Ometoato & 0.01 & 109.00 & 4.35 & 0.0012 \\
\hline Oxadixyl & 0.01 & 109.70 & 4.12 & 0.0011 \\
\hline Oxamil & 0.01 & 77.37 & 3.83 & 0.0007 \\
\hline
\end{tabular}


Table 1. Continued.

\begin{tabular}{|c|c|c|c|c|}
\hline Pesticide & LOQ & Recovery & SD\% & UM \\
\hline Oxasulfuron & 0.01 & 87.33 & 6.58 & 0.0014 \\
\hline Oxicarboxin & 0.01 & 78.08 & 4.12 & 0.0008 \\
\hline Paclobutrazol & 0.01 & 90.82 & 6.36 & 0.0014 \\
\hline ParaoxonEtil & 0.01 & 90.64 & 4.04 & 0.0009 \\
\hline ParaoxonMetil & 0.01 & 92.17 & 4.84 & 0.0011 \\
\hline Penconazol & 0.01 & 89.72 & 6.82 & 0.0015 \\
\hline Pencycuron & 0.01 & 85.63 & 6.18 & 0.0013 \\
\hline pendimentalina & 0.01 & 92.84 & 4.33 & 0.0010 \\
\hline Permetrina & 0.03 & 75.66 & 3.12 & 0.0029 \\
\hline Phentoato & 0.01 & 90.41 & 6.27 & 0.0014 \\
\hline Phorato & 0.01 & 94.47 & 5.85 & 0.0014 \\
\hline Phosalone & 0.01 & 81.96 & 6.90 & 0.0014 \\
\hline Phosfamidon & 0.01 & 91.08 & 4.64 & 0.0010 \\
\hline Phosmet & 0.01 & 93.78 & 4.62 & 0.0011 \\
\hline Picoxistrobin & 0.01 & 85.13 & 3.83 & 0.0008 \\
\hline PiperonilButoxide & 0.02 & 90.05 & 7.92 & 0.0018 \\
\hline Piraclostrobin & 0.02 & 86.11 & 10.11 & 0.0022 \\
\hline Pirazofos & 0.02 & 87.70 & 7.59 & 0.0016 \\
\hline Piridabem & 0.01 & 104.04 & 4.42 & 0.0011 \\
\hline Piridafention & 0.01 & 80.66 & 7.66 & 0.0015 \\
\hline pirimetanil & 0.01 & 115.30 & 4.55 & 0.0013 \\
\hline Pirimicarb & 0.01 & 91.07 & 4.64 & 0.0010 \\
\hline PirimifosEtil & 0.01 & 87.26 & 7.04 & 0.0015 \\
\hline PrimifosMetil & 0.01 & 86.15 & 5.75 & 0.0012 \\
\hline procloraz & 0.01 & 82.78 & 7.32 & 0.0015 \\
\hline Profenofos & 0.01 & 82.44 & 5.69 & 0.0012 \\
\hline promecarb & 0.01 & 92.82 & 3.69 & 0.0008 \\
\hline prometrin & 0.01 & 81.61 & 5.75 & 0.0012 \\
\hline Propargito & 0.01 & 86.03 & 5.95 & 0.0013 \\
\hline Propiconazol & 0.01 & 95.31 & 3.01 & 0.0007 \\
\hline propoxur & 0.01 & 86.93 & 5.26 & 0.0011 \\
\hline Protiofos & 0.01 & 88.49 & 5.95 & 0.0013 \\
\hline Pyretrins & 0.01 & 90.07 & 4.57 & 0.0010 \\
\hline Pyrifenox & 0.01 & 87.96 & 7.02 & 0.0015 \\
\hline Pyriproxifen & 0.01 & 77.62 & 3.18 & 0.0006 \\
\hline Simazina & 0.01 & 93.11 & 4.35 & 0.0010 \\
\hline Spirodiclofeno & 0.01 & 89.87 & 6.08 & 0.0014 \\
\hline Spiroxamine & 0.01 & 87.50 & 7.10 & 0.0015 \\
\hline Spnosad & 0.01 & 80.82 & 4.15 & 0.0008 \\
\hline SulfometuronMetil & 0.01 & 89.85 & 5.64 & 0.0013 \\
\hline Sulfotep & 0.01 & 93.42 & 6.11 & 0.0014 \\
\hline Sulprofos & 0.01 & 86.28 & 6.26 & 0.0013 \\
\hline tebuconazol & 0.02 & 102.06 & 9.66 & 0.0024 \\
\hline Tebufenozide & 0.01 & 81.97 & 6.96 & 0.0014 \\
\hline Tebufenpirad & 0.01 & 80.02 & 7.32 & 0.0015 \\
\hline Terbufos & 0.01 & 87.97 & 6.49 & 0.0014 \\
\hline Tetraconazol & 0.02 & 93.36 & 8.76 & 0.0020 \\
\hline Tetradifon & 0.01 & 84.54 & 3.65 & 0.0008 \\
\hline Thiacloprid & 0.01 & 85.31 & 6.54 & 0.0014 \\
\hline Thiometon & 0.01 & 86.53 & 7.04 & 0.0015 \\
\hline Thionazin & 0.01 & 98.07 & 4.77 & 0.0012 \\
\hline Tiabendazol & 0.01 & 81.40 & 6.62 & 0.0013 \\
\hline
\end{tabular}

Table 1. Continued.

\begin{tabular}{|l|l|l|l|l|}
\hline Pesticide & LOQ & Recovery & SD\% & UM \\
\hline Tiametoxam & 0.01 & 84.07 & 4.75 & 0.0010 \\
\hline Tiobencarb & 0.01 & 90.90 & 6.02 & 0.0014 \\
\hline Tiobencarb & 0.01 & 90.90 & 6.02 & 0.0014 \\
\hline Tiodicarb & 0.01 & 78.12 & 3.77 & 0.0007 \\
\hline TiofanatoMetil & 0.02 & 97.39 & 7.13 & 0.0017 \\
\hline Tolclofosmetil & 0.01 & 90.08 & 6.25 & 0.0014 \\
\hline Tolifluanid & 0.01 & 85.61 & 6.14 & 0.0013 \\
\hline triadimefon & 0.01 & 89.35 & 6.98 & 0.0015 \\
\hline Triadimenol & 0.02 & 92.27 & 7.17 & 0.0016 \\
\hline Triazofos & 0.01 & 86.20 & 6.17 & 0.0013 \\
\hline Triclorfon & 0.01 & 83.62 & 5.52 & 0.0011 \\
\hline Tricyclazol & 0.01 & 80.28 & 3.20 & 0.0006 \\
\hline Tridemorf & 0.01 & 87.59 & 7.07 & 0.0015 \\
\hline Triflimizole & 0.01 & 87.35 & 6.86 & 0.0015 \\
\hline Trifloxitrobim & 0.01 & 86.30 & 7.15 & 0.0015 \\
\hline Triflumuron & 0.01 & 89.89 & 4.15 & 0.0009 \\
\hline Triticonazol & 0.01 & 90.50 & 6.72 & 0.0015 \\
\hline Vamidation & 0.00 & 73.20 & 2.21 & 0.0004 \\
\hline
\end{tabular}

\section{References}

[1] European Commission Regulation (EC), "No. 396/2005 of 23 February 2005 on maximum residue levels of pesticides in or on food and feed of plant and animal origin and amending Council Directive 91/414/EEC," Official Journal of the European Union, vol. L70, pp. 1-16

[2] Document No. SANCO/12495/2011, Method Validation and Quality Control Procedures for Pesticide Residues Analysis in Food and Feed, http:// ec.europa.eu/food/plant/protection/ pesticides/docs/qualcontrol_en.pdf 